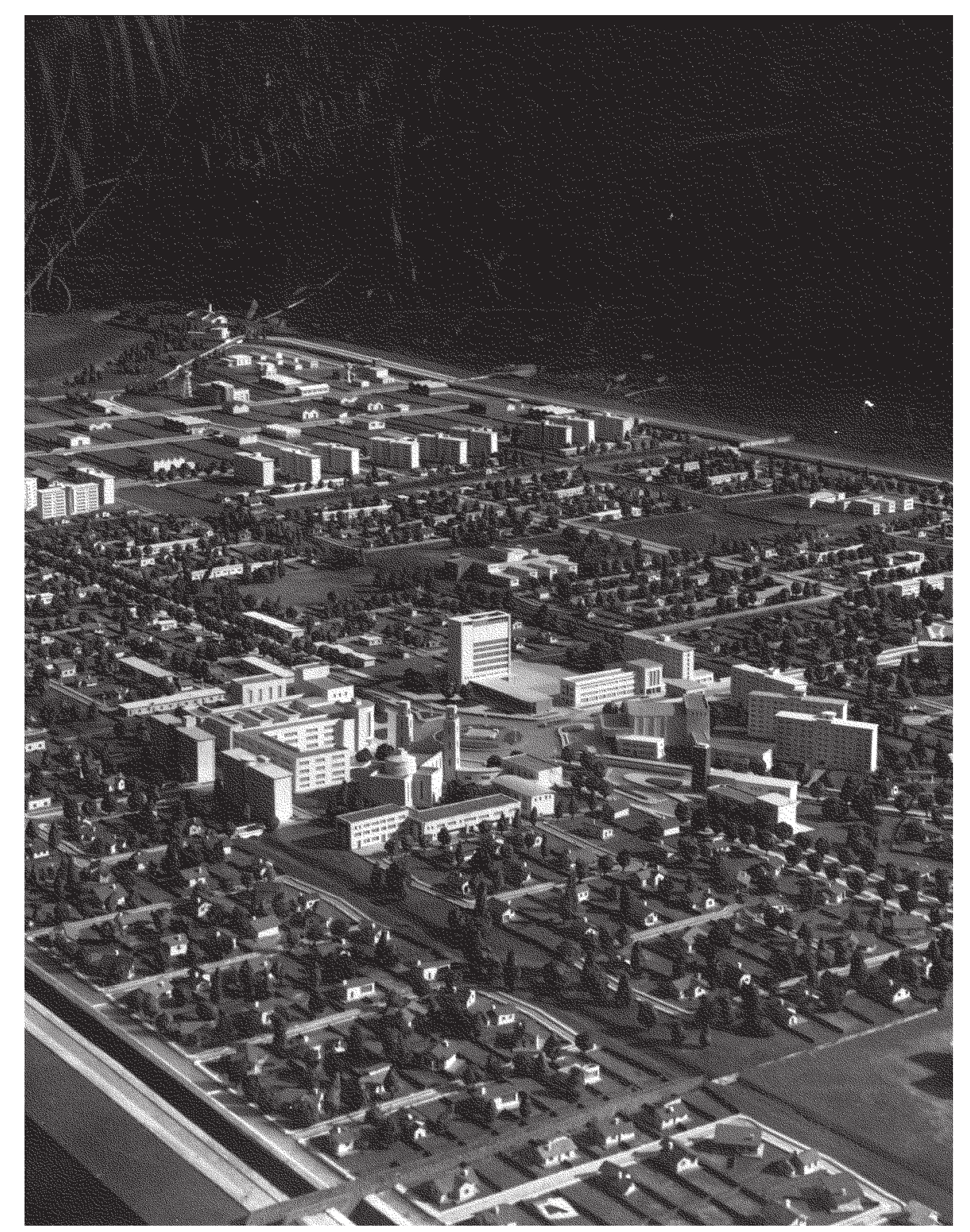



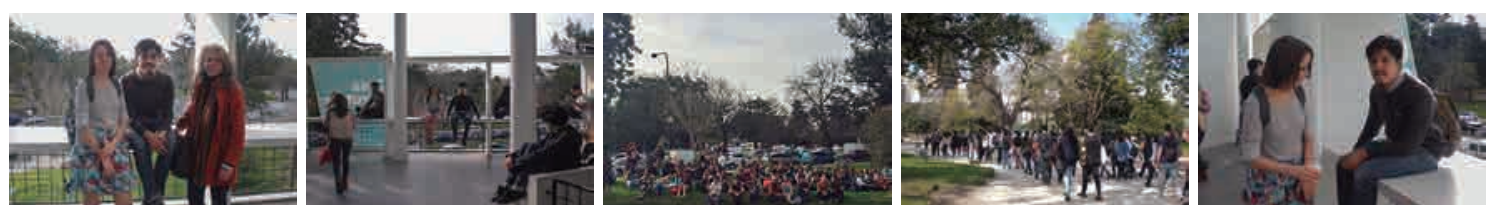

Fotos: Diego Fernando Machin

\title{
DEL URBANISMO AL PLANEAMIENTO: PASTOR EN LA REVISTA DE ARQUITECTURA (1943-1951)'
}

\author{
FROM URBAN PLANNING TO PLANNING: PASTOR IN LA REVISTA DE \\ ARQUITECTURA (1943-1951)
}

\section{Juan José Gutiérrez²}

RESUMEN

\begin{abstract}
El urbanismo como disciplina se consolida en la Argentina en la primera mitad del siglo XX. Se institucionaliza en la academia mediante la estructura de las carreras y los institutos de investigación. En paralelo a este proceso logra insertarse en la burocracia estatal obteniendo así una mayor capacidad de operar en la ciudad. Su desarrollo como disciplina teórica y política pública específica se realiza a mediados del siglo pasado con el nombre de "planeamiento". Las discusiones dentro de la estructura del Estado, la academia, las asociaciones profesionales y el espacio público encuentran un nodo discursivo en la Revista de Arquitectura de la Sociedad Central de Arquitectos. Dentro de esta fuente será sobresaliente, en la década de 1940, el lugar del arquitecto José Manuel Felipe Pastor, como teórico del planeamiento, y su utilización de la teoría del urbanismo inglés y americano. El siguiente trabajo nos permitirá explorar como se construyó el discurso que actualizó el urbanismo, a través de los escritos de Pastor en la mencionada Revista de Arquitectura.
\end{abstract}

Palabras clave: arquitectura, urbanismo, planificación, revistas, vivienda de interés social.

\section{ABSTRACT}

Urban planning as a discipline was consolidated in Argentina in the first half of the twentieth century. It was institutionalized in academia through the establishment of courses of undergraduate and graduate study and through research institutes. At the same time this process was taking place, urban planning succeeded in inserting itself into the state bureaucracy, thereby gaining a greater ability to operate in the city. Its development as a theoretical discipline and specific public policy occurred during the middle of the last century under the name "planning". Discussions within the state structure, academia, professional associations and the public sphere found a discursive node in the Revista de Arquitectura de la Sociedad Central de Arquitectos (Architectural Journal of the Central Society of Architects). Within this source, in the decade of the 1940s the architect José Manuel Felipe Pastor stands out as a planning theorist and for his use of English and American urban planning theory. This article explores how the discourse that updated urban planning was constructed through Pastor's writings in Revista de Arquitectura.

Keywords: architecture, urban planning, planning, journals, social housing.

Artículo recibido el 8 de agosto de 2016 y aceptado el 18 de noviembre de 2016 DOI: https://doi.org/10.22320/07196466.2016.34.050.05

[1] Este artículo forma parte de los resultados de investigación de la Beca Maestría UBACyT, 2015-2017, otorgada por la Universidad de Buenos Aires al arquitecto Juan José Gutiérrez.

[2] Centro de Investigaciones de Historia de la Vivienda en América Latina - Instituto de la Espacialidad Humana - Facultad de Arquitectura, Diseño y Urbanismo - Universidad de Buenos Aires. tafgor@hotmail.com 


\section{INTRODUCCIÓN}

El afianzamiento del urbanismo como disciplina en la Argentina se puede situar en el año 1947 con la creación de la Facultad de Arquitectura y Urbanismo de la Universidad de Buenos Aires. Es la primera experiencia autónoma de la enseñanza de la disciplina que cuenta con independencia de la carrera de Ingeniería y de la Facultad de Ciencias Exactas, Físicas y Naturales (FCEFN). Incluye la incumbencia de urbanista junto con el título de Arquitecto. Antecedente de esto es la cátedra de urbanismo que se crea en 1933 en la Escuela de Arquitectura, pero aún dentro de la FCEFN. También fueron aportes a este proceso los títulos de posgrado con la especificidad de Urbanismo en dicha facultad y la creación del Instituto Superior de Urbanismo, ambos en el año 1948. Antes de la creación de estas instituciones, los arquitectos y los ingenieros ocupaban el rol de especialistas en urbanismo como poseedores del saber específico que les otorgaba un lugar jerárquico en una disciplina que contaba con aportes de la sociología, la geografía, el derecho y la geología.

En la década de 1940, el urbanismo adopta una metodología específica que lleva a llamarlo "planeamiento". Este cambio implicará un viraje en la forma de proyectar de los urbanistas (Novick y Piccioni, 1998:114)³ y así también un viraje de las políticas urbanas, como expresión derivada del saber teórico. Desde la discusión teórica será central la figura del arquitecto José Manuel Felipe Pastor como agente intelectual en el posicionamiento del planeamiento como actualización del urbanismo. 


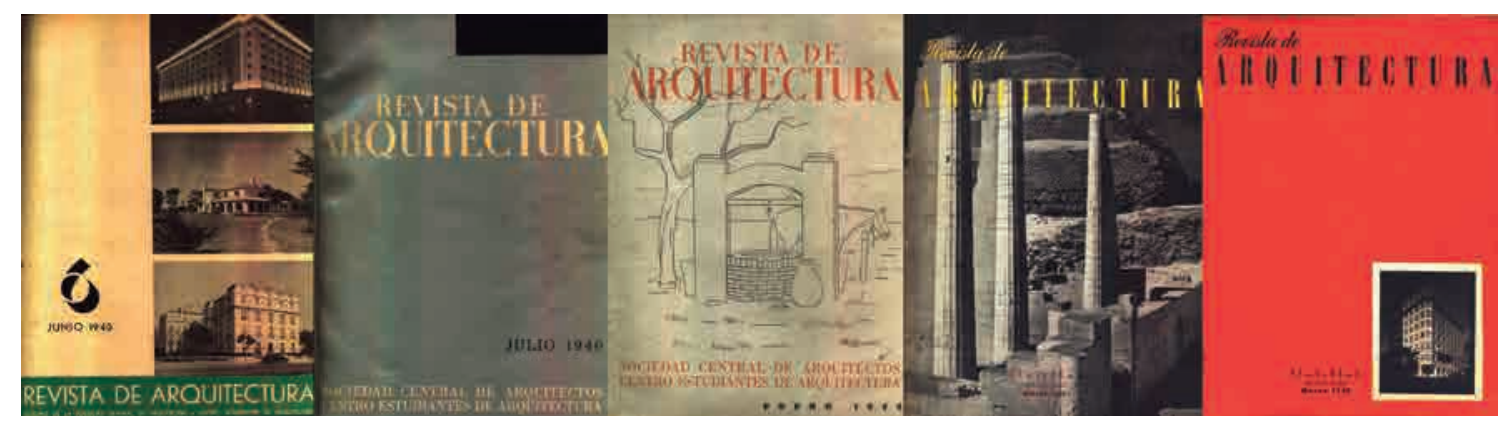

Figura 1 Revista de Arquitectura. Cambios gráficos en las tapas: junio 1940, julio 1940, enero 1944, marzo 1947, marzo 1948. Fuente: Revista de Arquitectura, 1940, pp. 193-194.

\section{METODOLOGÍA}

Esta investigación propone un primer avance en el estudio del posicionamiento del planeamiento, mediante la exposición de los escritos de Pastor en la Revista de Arquitectura (RdeA) de la Sociedad Central de Arquitectos. Este corpus bibliográfico permitirá estudiar como fue el proceso discursivo que opera en la disciplina del urbanismo, llevándola a su versión particular del planeamiento. El trabajar a partir de la metodología biografía profesional (Bruno, 2006) ${ }^{4}$ posibilita especificar conceptos tales como planeamiento. Pastor concibe como "planeamiento" una manera específica de operar en la ciudad que se presenta como el sumun de la democracia, como la humanización del urbanismo (1950: 47-48). Su definición de planeamiento incluye un esfuerzo no solo propositivo sino de divulgación, el cual ha de "reportar resultados positivos para la creación y consolidación de una conciencia popular del planeamiento" ("Divulgación del urbanismo y la arquitectura", 1949) ${ }^{5}$. Sus textos, tanto artículos como libros, participan del proyecto ya que operan no en la materia-ciudad, sino en los ciudadanos, cuyo consenso (o conciencia popular del planeamiento) es imprescindible para el diseño, la ejecución y el sostenimiento de los planes urbanos.

Se realizó así un exhaustivo rastreo de todas sus publicaciones en la Revista. A continuación, se enumeraron todos sus artículos, que reúnen distintas temáticas, distintas maneras de firmar y fueron elaborados bajo los distintos cargos que Pastor ocupó en la revista, con el fin de periodizarlos y dotarlos de mayor sentido. Así, se presenta la participación de Pastor en la RdeA en cuatro periodos: como colaborador externo, como colaborador especialista en vivienda social, como Secretario de Redacción y como Director. Una vez entendidos sus diferentes roles en la Revista, y el tono de sus artículos en dichos periodos, se construye una segunda periodización que divide la producción de sus artículos en dos: una etapa, en la que los textos se abocan al urbanismo, en términos generales, y otra en la que se habla específicamente de planeamiento.

\section{LA REVISTA DE ARQUITECTURA DE LA SOCIEDAD CEN- TRAL DE ARQUITECTOS}

"REVISTA DE ARQUITECTURA" ya no es la publicación de los estudiantes, expresión seria o risueña de sus inquietudes, ni la de un grupo que comienza sus luchas, sino la de una profesión que está adquiriendo a pasos agigantados su mayoría de edad y la consideración merecida en el concierto de las otras que han tenido la suerte de nacer antes. Debe estar al día. Debe llegar a ser la caja de resonancia del movimiento arquitectónico del país, interpretarlo, reflejarlo, y si se nos permitiere un quijotismo, expresión de un deseo que debe materializarse en la futura superación de nuestra magra capacidad, diríamos derechamente: orientarlo. ("Revista de Arquitectura cumple 25 años", 1940: 193-194).

La Revista de Arquitectura (Figura 1) fue fundada en 1915 por el Centro de Estudiantes de Arquitectura (CEA), perteneciente a la entonces Escuela de Arquitectura de

[4] La propuesta de construcción narrativa, que mediante el estudio del personaje estudia la disciplina, es heredera tanto del trabajo ya citado de Novick y Piccioni, como de estudios de biografía profesional, por ejemplo, los Ilevados a cabo por Paula Bruno sobre Paul Groussac.

[5] La cita hace referencia a una entrada de la sección Crónica, sin número de página ni firma, que pertenece al periodo de Pastor como Director de la RdeA. 
la FCFEN (antecedente directo de la FADU UBA actual). La creación de la Revista tuvo como fundamento el posicionar la arquitectura entre las disciplinas reconocidas, especialmente dentro de una puja con ingeniería, con la cual compartía el espacio en la facultad. De hecho, el CEA no era reconocido como tal por la Federación Universitaria de Buenos Aires, ya que el estatuto de la universidad establecía que solo podía haber un centro de estudiantes por facultad (Cirvini 2011: 34-35) y ya existía previamente el centro de estudiantes de ingeniería.

En el año 1917, la Sociedad Central de Arquitectos comenzó a formar parte de la Revista. En 1923, luego de una fase de irregularidad, se creó un Comité Directivo donde se posicionó como protagonista la SCA. Este cambio trajo regularidad editorial, incremento en publicidades de empresas ligadas a la producción y la pérdida de cierto carácter polémico de las publicaciones (Gentile, 2004).

En el periodo comprendido entre 1933 y 1943, la revista abordó principalmente dos temáticas (Figura 2). Por una parte, se enfocó hacia las estéticas modernas y las nuevas tecnologías; abundan entonces artículos sobre cocinas eléctricas, acondicionamiento de aires, calefacción iluminación, refrigeración, obras sanitarias, azoteas impermeables, $\mathrm{H}^{\circ} \mathrm{A}^{\circ}$ y acero, vinculándose así fuertemente a los sectores de la industria de la construcción. Y, por otra parte, aparecen también numerosas editoriales que intentan establecer la institución del urbanismo como práctica consagrada y específica de los arquitectos, así como lo muestra la creación en 1933 de la cátedra de Urbanismo en la Escuela de Arquitectura, a instancias de Coni Molina, ex presidente de la SCA (ídem).

El año 1943 finaliza con Federico de Achával como Director de la Revista. Bajo su dirección, ésta toma un enfoque nacionalista y de tinte conservador católico, que se ve matizado con los aportes de Pastor, Cappagli y Moller (siendo el primero quien lo reemplaza en la dirección, en julio de 1947). Ahora bien, Achával vira dicha tendencia, tal vez influenciado por la derrota del fascismo en Europa y la poca resonancia de su mensaje en el medio local. De esa forma, incluye distintas corrientes modernas internacionales, una de ellas será el planeamiento de la mano de Pastor. Este incorpora en su propuesta gran parte de la experiencia en materia de gestión urbana, de políticas de vivienda y el planeamiento inglés de posguerra (Gutiérrez, 1993). Dentro de lo relativo a lo nacional, habrá tres tópicos centrales: la vivienda social, la reconstrucción de San Juan y la relación entre el planeamiento y el Estado. Este periodo finaliza con tres entregas en el año 1951 (enero, noviembre y diciembre, la primera con Pastor como presidente y luego Álvarez ocupando dicho rol). A partir de este año, la revista ya no cuenta con la participación del CEA, en paralelo a ello desaparecerán los artículos sobre trabajos de estudiantes y se reforzará la relación de la revista con el gobierno peronista en tanto ésta publica con tono favorable obras llevadas a cabo por el gobierno, como la Fundación Eva Perón, el Plan Quinquenal y el Barrio Presidente Perón. Una clara diferencia con la postura ambigua del periodo anterior, que algunos autores coinciden en caracterizar como una resistencia cultural (Cirvini, 2011: 44), en la medida en que la revista no hablaba de política.

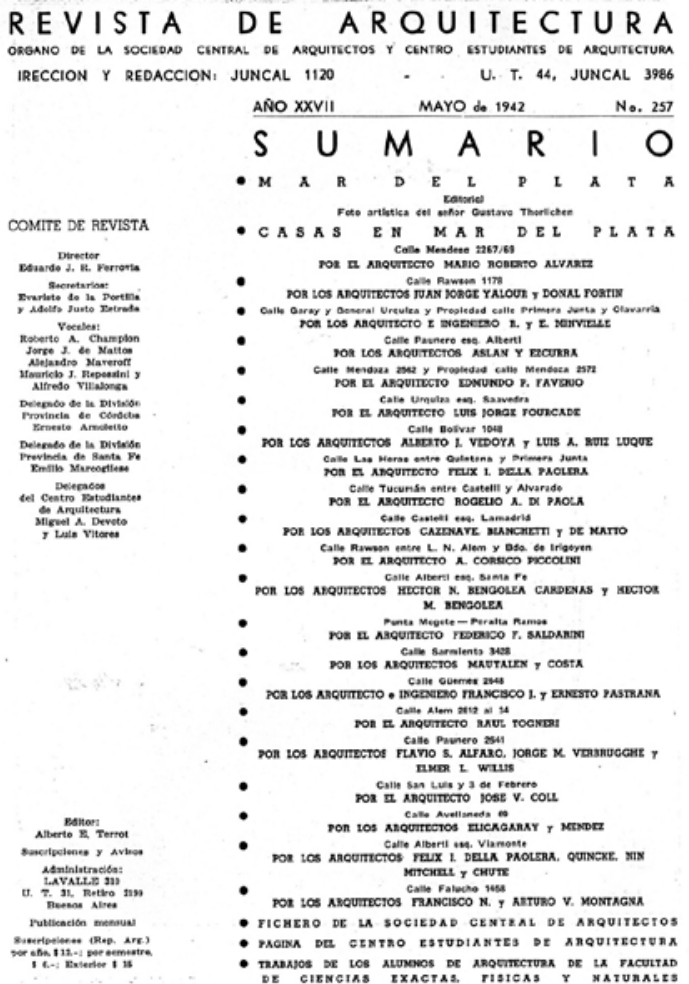

Figura 2 La Revista de Arquitectura como muestrario de obras. Sumario de mayo de 1942. Fuente: Revista de Arquitectura $1942, n^{\circ} 257$, p. 195

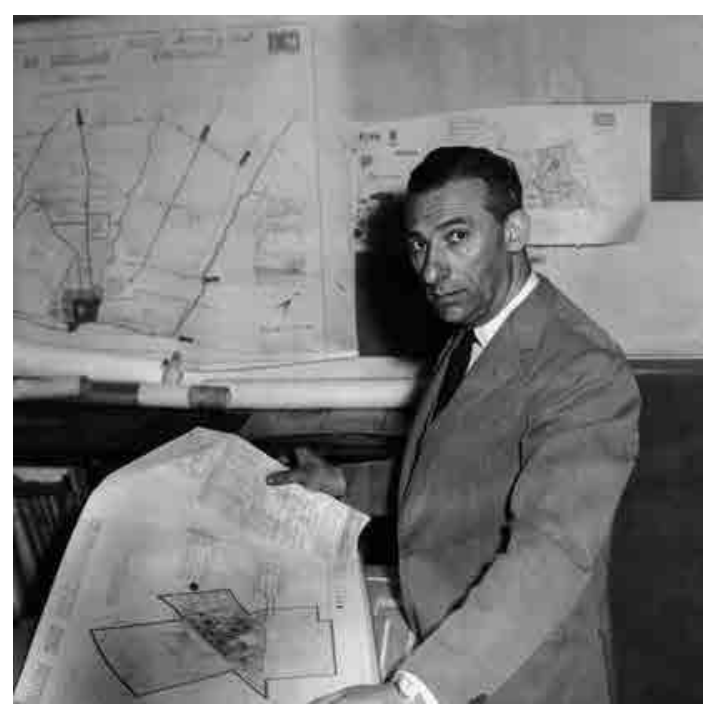

Figura 3 José Manuel Felipe Pastor, 1960. Fuente: Colección Pastor, Archivo del Instituto de Arte Americano FADU-UBA. 


\section{PASTOR EN LA REVISTA DE ARQUITECTURA SCA}

El arquitecto especialista en urbanismo (tal como firmaba), José Manuel Felipe Pastor (Figura 3), nació en 1914, en Corrientes, Argentina. Egresado de la Escuela de Arquitectura de la Facultad de Ciencias Exactas, Físicas y Naturales de la Universidad de Buenos Aires en 1944, tuvo una fuerte participación en la construcción del planeamiento argentino mediante la importación de teorías y experiencias británicas y americanas (Gorelik, 2004: 46-47). La trayectoria de Pastor implica un aporte al planeamiento argentino, en tres cuerpos bien marcados:

Participación institucional: en la que resaltan su colaboración en el Consejo de Reconstrucción de San Juan (1948-1952) y una inserción institucional en el Gobierno de la Provincia de Buenos Aires en la Comisión Asesora del Gran Buenos Aires (1948-1950).

Proyectos reguladores: sobre todo desde el Instituto de Planeamiento Regional y Urbano (IPRU) cofundado con el ingeniero José Bonilla y el arquitecto Alfredo Etcheverry en 1952.

Divulgación: como promoción del planeamiento y sobre la necesidad de la construcción de un Plan Urbano y Rural Nacional desde el IPRU, desde su extensa bibliografía y -lo que constituye el centro de este artículo- desde numerosas publicaciones en la Revista de Arquitectura de la Sociedad Central de Arquitectos (RdeA) y en Nuestra Arquitectura (NA $)^{6}$

La Revista de Arquitectura (1915 - a la actualidad) es la publicación especializada de mayor trayectoria en la Argentina (Cirvini, 2011: 35). La figura de Pastor se inserta en ella como novedad a través de ensayos sobre vivienda social y, más tarde, sobre urbanismo. Dicha producción contempla más de cincuenta artículos, publicados entre 1944 y 1951, cuyo estudio merece especial atención al ocupar Pastor distintos lugares jerárquicos dentro de la revista. Su primer artículo constituye un trabajo sobre arquitectura social y económica (Pastor, 1944). Ahora bien, vale destacar que en esos años la revista presenta dos trabajos producidos por Pastor y Prats para el Salón Nacional de Arquitectura (Figura 4). El primero de ellos, "Una posta" (1943), es un escrito anónimo que presenta el trabajo de Pastor y Prats dentro de la categoría de Arquitectura Social. El proyecto propone todo un sistema de colonización mediante hoteles, villas turísticas y campings; en éste ya aparecen temáticas como el planeamiento, la dualidad máquina-hombre, el fuerte acento en la variable económica y la importancia de la construcción de instrumentos públicos de urbanismo. Por su parte, en "Centro Juvenil de Recreación" (Pastor y Prats, 1944) se amplían los temas abordados en el trabajo anterior, incluyendo la variables de "conquista social", se suma un tono moral y se prevén los cambios que provocará la post-guerra. De esta manera, estos dos trabajos adelantan el perfil que Pastor ocupara en la revista. Incluye este breve periodo -de Pastor como colaborador externo- La futura San Juan, el primer texto de un extenso grupo de artículos sobre la reconstrucción de esa ciudad, el cual es firmado por Pastor con el seudónimo de "Urbanalista", el que volverá a usar seguidas veces.

A partir de un segundo artículo, firmado como Pastor, pasa a participar formalmente de la nómina de colaboradores de la Revista, en marzo de 1944. Desde esa fecha a septiembre del mismo año, presenta 5 artículos sobre arquitectura social $y$, en concreto, en el número de mayo, su artículo "La vivienda social" lleva como subtítulo "Estudio del Arq. José M. F. Pastor, colaborador permanente, encargado de nuestra sección Arquitectura Social", que explicita el perfil de Pastor en sus primeras participaciones en la RdeA.

Desde enero de 1945 hasta mayo de 1947 se presenta la etapa de Pastor como Secretario de Redacción. Por entonces, firma 7 artículos como Pastor, 3 como Urbanalista y otros 3 como "Redacción". El eje en la vivienda, su economía y carácter popular, deja lugar al planeamiento $y$ al referente inglés, en primer lugar, y americano, en segundo. Como línea transversal a estos dos tópicos, se encuentran las discusiones sobre legislación. Títulos como Vivienda popular, subsidios e iniciativa privada; Bases del planeamiento británico; Códigos regionales en lugar de códigos municipales de edificación; Un año de la legislación urbanística en el Perú7; El planeamiento urbano en la nueva Francia, evidencian la temática y especifican las referencias. No solo se expone el urbanismo sino que se postula su pertinencia en la órbita nacional. No solo presenta experiencias, sino que propone políticas metodológicas y exhibe sus resultados siempre positivos, siempre aplicables, siempre explícitamente necesarios.

Posicionado dentro de la estructura jerárquica de la Revista, entendiendo esto como la posibilidad de tomar decisiones sobre lo que se publica y lo que no, se puede incluir, en el recorte relativo a esta investigación, artículos que exceden a su autoría. Así, se pueden admitir otros 18 artículos de autores varios que continúan la discusión del planeamiento como una necesidad nacional.

La última etapa de Pastor en la RdeA comienza en julio de 1947, cuando empieza a ejercer como Director de la revista. Su producción directa consta de 29 artículos. Firma como Pastor 5 de ellos y otros 5 como Urbanalista. Toma suma importancia aquí el tópico de la reconstrucción de San Juan (Rigotti, 2014) (Figura 5). A su vez, 19 artículos, firmados como "Dirección", son de tópico variado: concursos, vivienda popular y planeamiento, problemas profesionales, tecnología. Esta selección incluye además textos con la clara firma de Pastor, en los cuales se critica la expresión "ciudades jardinadas"

[6] A pesar de tener un mayor énfasis en la temática del planeamiento, las publicaciones en la revista Nuestra Arquitectura no han sido consideradas en este estudio, debido a que Pastor no ocupa allí un lugar institucional. Son veinte los artículos que Pastor publica en esta revista entre 1945 y 1951

[7] A fin a la referencia es la participación de Pastor, desde 1947, como miembro honorífico del Instituto de Urbanismo del Perú y su carácter de profesor invitado en la Universidad de Cusco en 1956. 


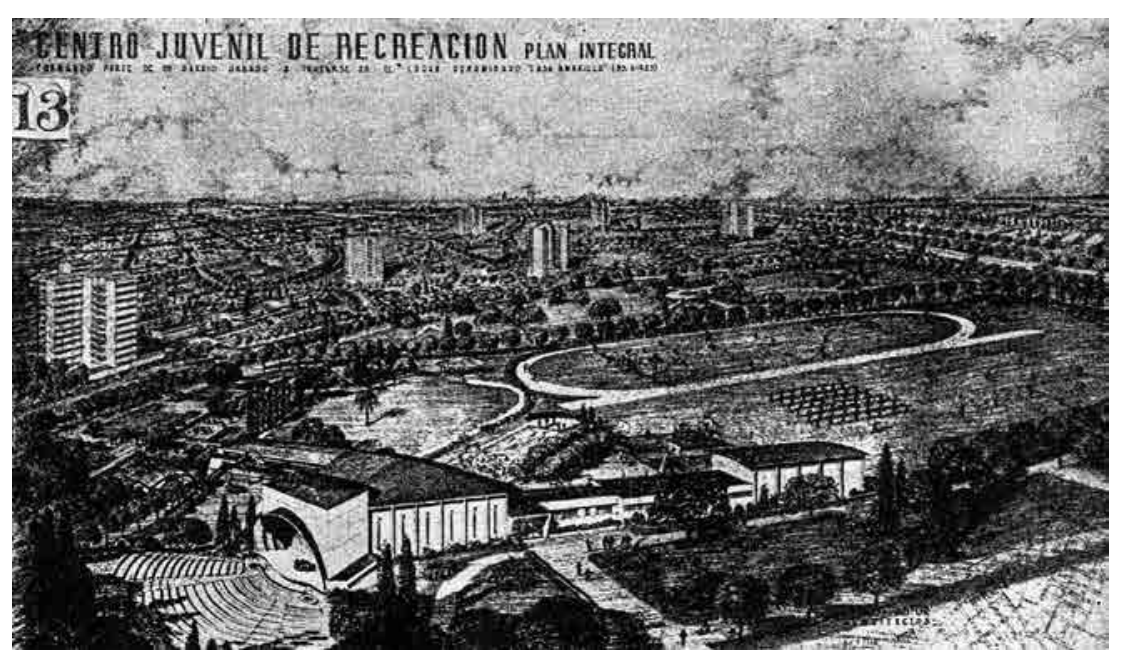

Figura 4 Centro Juvenil de Recreación: Plan integral, mención especial en el V Salón Nacional de Arquitectura, Prats y Pastor. Fuente: Revista de Arquitectura, 1944, n²77, p. 39

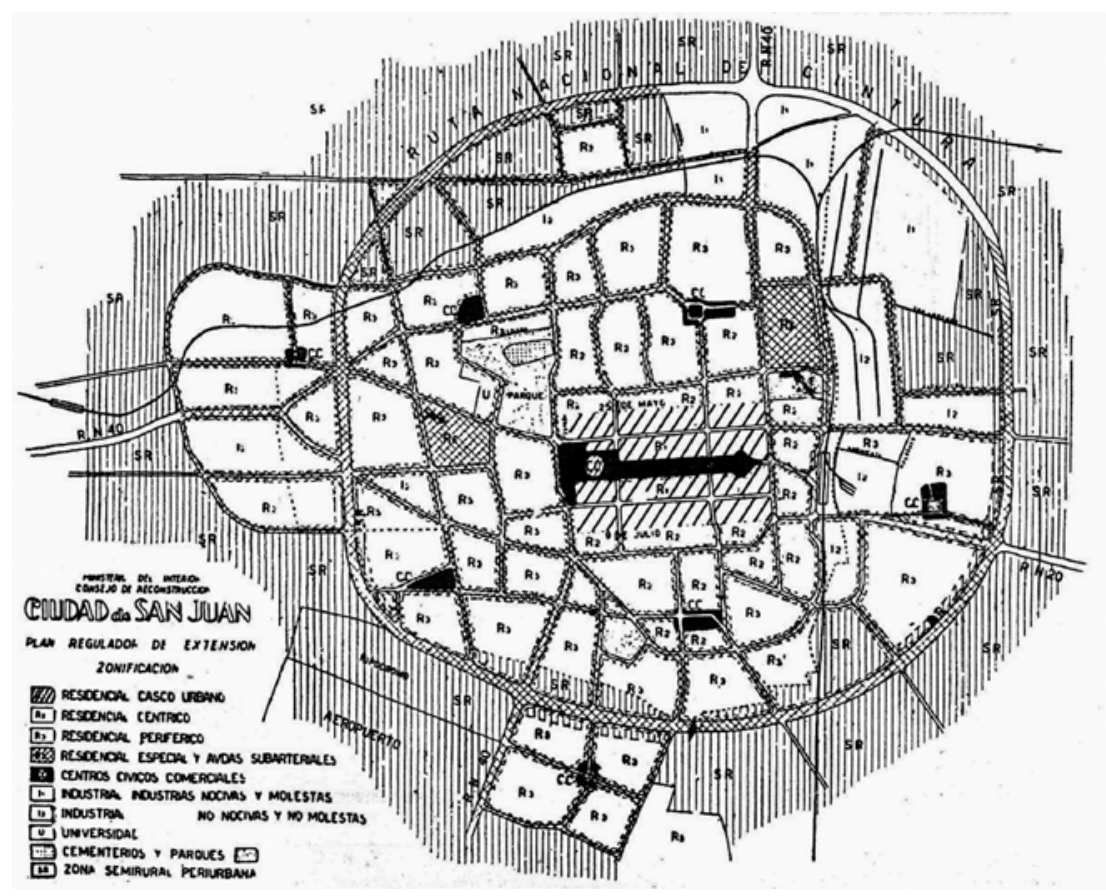

Figura 5 Plano para la reconstrucción de San Juan. Fuente: Revista de Arquitectura, 1950, n³55, p. 220. 
por constituir un intento explícito de homologarse con la de "ciudad-jardín". Es exclusividad de esta etapa la aparición de artículos con esa firma, al igual que lo fue en el caso de aquellos firmados como "Redacción" en el periodo anterior. Rescatar algunos artículos en particular, como "Justa ponderación del urbanismo lecorbusierano"; "El Gran Buenos Aires"; "El plan regulador de Buenos Aires"; "Ciudades jardín del presente"; "Ciudades jardín del futuro"; "Crece la conciencia popular de planeamiento"; $y$ "Promesa y realidad en materia de planeamiento", permite evidenciar la discusión que Pastor propone en referencia al posicionamiento del planeamiento en la práctica urbanista.

Imitando el mecanismo de recorte del anterior periodo, en el último grupo de textos se encuentra 80 artículos referentes al planeamiento, que van desde la importancia de éste para la vivienda popular hasta su valor en la construcción de la familia católica. Es destacable la notoriedad que toma la sección "Crónica", la que incluye 21 artículos de los 80 antes mencionados, y se halla cargada tanto de noticias afines como de editoriales. Asimismo, adquieren relevancia algunos referentes como Poëte y Howard y la fuerte intención de situar en la agenda el Día del Urbanismo (impulsado por Della Paolera).

Luego de la edición de enero de 1951, donde Pastor aún figura como Director, se pierde la regularidad de la Revista. El próximo tomo se editará en noviembre del mismo año, ya con la dirección de Raúl J. Álvarez.

\section{DEL URBANISMO AL PLANEAMIENTO}

Como se señaló previamente, luego de abordar la producción toda de Pastor, se hace necesario realizar una segunda periodización del cuerpo de artículos ya citados. Aquí se subraya el punto de quiebre en el proceso de construcción discursiva del concepto de planeamiento. Para ello, rescatamos una cita textual de Mazza y Bruno: “Entre 1940 y 1944 se publican en la Revista de Arquitectura alrededor de 14 artículos. Aproximadamente igual cantidad se publica entre 1944 y 1946, sumándose al menos dos libros de autoría del arquitecto Pastor: San Juan Piedra de Toque del Planeamiento Nacional y Urbanismo con Planeamiento" (2008: 9).

En el periodo 1940-1944 se encuentran los siguientes artículos referidos al urbanismo:

1940, enero: “La segunda exposición municipal de urbanismo".

1940, marzo: "La evolución de Buenos Aires en 50 años".

1940, marzo: "El urbanismo. La ciudad orgánica".

1940, abril: "El nuevo criterio en el amanzanamiento de las tierras urbanas", por Bereterbide.

1940, abril: "Ciclo de conversaciones sobre urbanismo y vivienda popular".

1940, agosto: "Acerca de la obra Manual de urbanismo de Karl H. Brunner".
1940, octubre: "Congreso de la población".

1940, noviembre: "La enseñanza del urbanismo en las universidades de los Estados Unidos".

1940, diciembre: "Los pueblos Greenbelt en los Estados Unidos".

1942, julio: “Qué quiere decir Instituto de Urbanismo de la Facultad de Arquitectura?"

1942, noviembre: "La ciudad de Buenos Aires, ha crecido con empuje y desorden juvenil..."

1942, noviembre: "El catastro de la ciudad de Buenos Aires".

1942, noviembre: "Ejecución del catastro de la ciudad de Buenos Aires y su función económica, social y urbana".

1943, enero: “El plan Haussman", por Robert Moses.

1943, diciembre: "Trabajos premiados" (expone "Barrio vivienda para clase media" y "Un centro de turismo para la clase media").

Este primer conjunto de textos es en poco comparable con el grupo siguiente. En ese segundo grupo, correspondiente a la producción de entre 1944 y 1946, se podría incluir el primer y segundo periodo de Pastor, desde el punto de vista de su participación institucional en la RdeA. Si en términos cuantitativos existe una similitud, la observación de los temas abordados, que se realiza a continuación, vuelve imposible la comparación.

El contenido del conjunto que comienza con los artículos de 1940 y termina al comenzar 1944 (el año del primer artículo de Pastor en la Revista) podría ser resumido como noticias de eventos institucionales (congresos, inauguración de instituto) y reseñas (becarios en el exterior o publicaciones recientes), donde lo que prima es el urbanismo de la forma. Este último punto lo evidencia "La segunda exposición municipal de urbanismo" (Figura 6) donde los trabajos expuestos son proyectos urbanos o propuestas de normativas de restricción de dominio. En las antípodas se encuentra el segundo conjunto donde, desde el comienzo, "Una posta" propone el camino inverso. No expone al urbanismo como la disciplina de la forma sino que, con un proyecto de arquitectura, esboza un programa a escala regional que pone el esparcimiento como un derecho social de la nueva clase media en pleno proceso de desarrollo. Es anecdótico que este reconocimiento a un proyecto de Pastor y Prats sea publicado justamente en enero, pero más allá de lo oportuno de la fecha, ello marca una nueva etapa de la Revista donde el proyecto urbano es reemplazado por el plan.

A pesar que este proceso presenta un claro día de comienzo, no se dará de forma tajante. Tendrá un desarrollo en el cual la cuestión social de la vivienda obrera virará de ser un reclamo en base a un déficit, hacia ser un punto dentro de una propuesta íntegra, dentro de un plan. Retomando la cita de Mazza, este proceso de viraje se evidencia en los libros San Juan Piedra de Toque del Planeamiento Nacional (1945) y Urbanismo con Planeamiento (1947), pero también en Curso básico de planeamiento urbano y rural, que mal no se incluye 


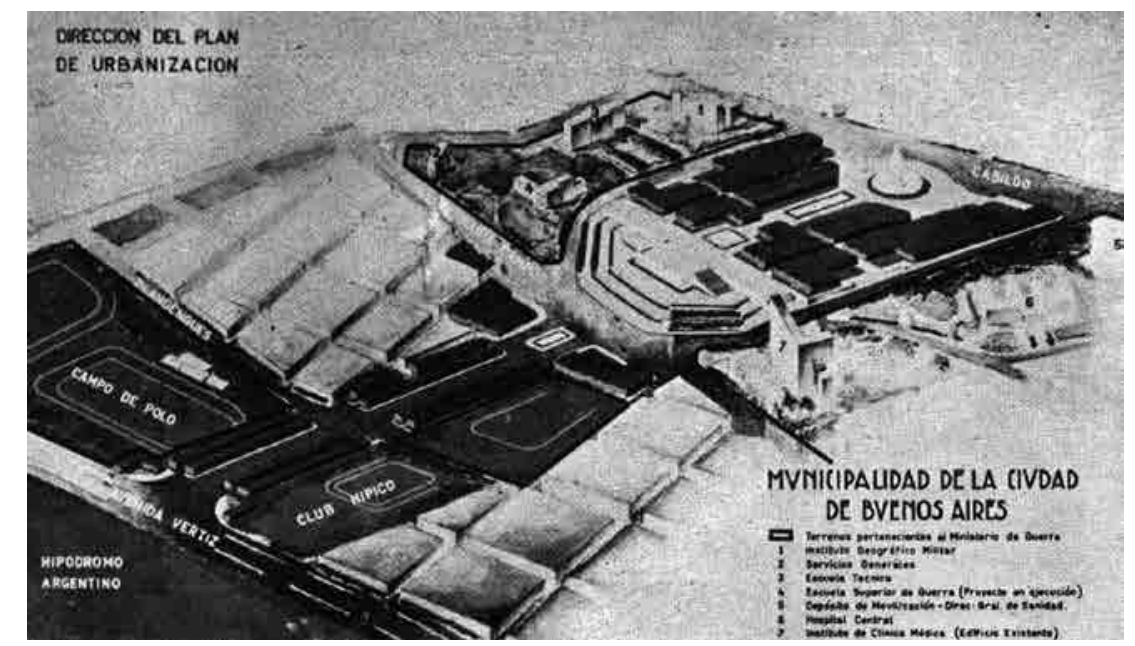

Figura 6 Lámina expuesta en la Segunda Exposición Municipal de Urbanismo. Fuente: Revista de Arquitectura de enero de 1940

en esta consideración por haber sido editado en 1950 aun cuando constituye la transcripción de unas clases dictadas por Pastor en el año 1947. Este conjunto de textos reúne la discusión de la vivienda obrera; es una verdadera demanda por posicionar al planeamiento como único medio para conseguir esa estructura que construirá la causa común.

En abril de 1944 debuta en RdeA la sección "Crónica", que reemplaza "Noticias de la SCdeA", título bajo el cual aparecieron artículos en varios números de la Revista. El segmento se ubica al principio y al final, ocupando espacios reducidos entre los anuncios publicitarios. En un principio, esta sección será reservada para noticias de agenda: cambios de gerencias en delegaciones gubernamentales, noticias del exterior, cambios del código de edificación, pero rápidamente comenzará a dar lugar a editoriales anónimas sobre vivienda y a críticas a instituciones gubernamentales, como las noticias sobre la reconstrucción de San Juan y evaluaciones sobre la Administración de la Vivienda o el Banco Hipotecario Nacional. Es destacable como Pastor utiliza este espacio a partir del mismo ejemplar en el que comienza a ejercer como Director de la Revista. Escribe, en junio de 1947, "Alrededor del problema de la vivienda en Buenos Aires", firmando como Urbanalista, y "Arquitectos argentinos durante la dominación hispánica", firmando como Pastor. Este número incluye, también dentro del segmento Crónica, dos artículos sobre planeamiento, de La Plata y de Gran Bretaña. Crónica será un espacio donde hará presencia constante el Día del Urbanismo como intento de colocar en la agenda nacional e internacional dicha celebración.
Este último periodo se ve determinado por los cargos de jerarquía que Pastor ocupa en la revista (Secretario de Redacción y Director). Aquí se puede identificar tres grupos de textos. El primero conformado por los ya nombrados artículos de Pastor, que se centran en la necesidad del plan. Allí, la ciudad-jardín es la protagonista principal, y tres artículos fundamentan ese rol: "Ciudades jardín del presente"; "Ciudades jardín del futuro" y "Ciudad-jardín "El Libertador"'. Esta triada de textos tiene como ejes el referente extranjero, la concreción nacional y el prototipo a replicar, respectivamente. El segundo conjunto corresponde al espacio de Crónica donde 40 de los 43 números del periodo presentan noticias o proyectos sobre la institucionalización del planeamiento y editoriales sobre la problemática de la vivienda y las deficiencias de las políticas de planeamiento. Centrales, en estos términos, son artículos como "Divulgación del urbanismo y la arquitectura" (octubre de 1949), donde la noticia (la inauguración de una revista de arquitectura de la Facultad de Arquitectura y Urbanismo y la divulgación del film del Plan para el Bajo Belgrano) se escribe como un avance concreto del planeamiento en el espacio de la opinión pública. 

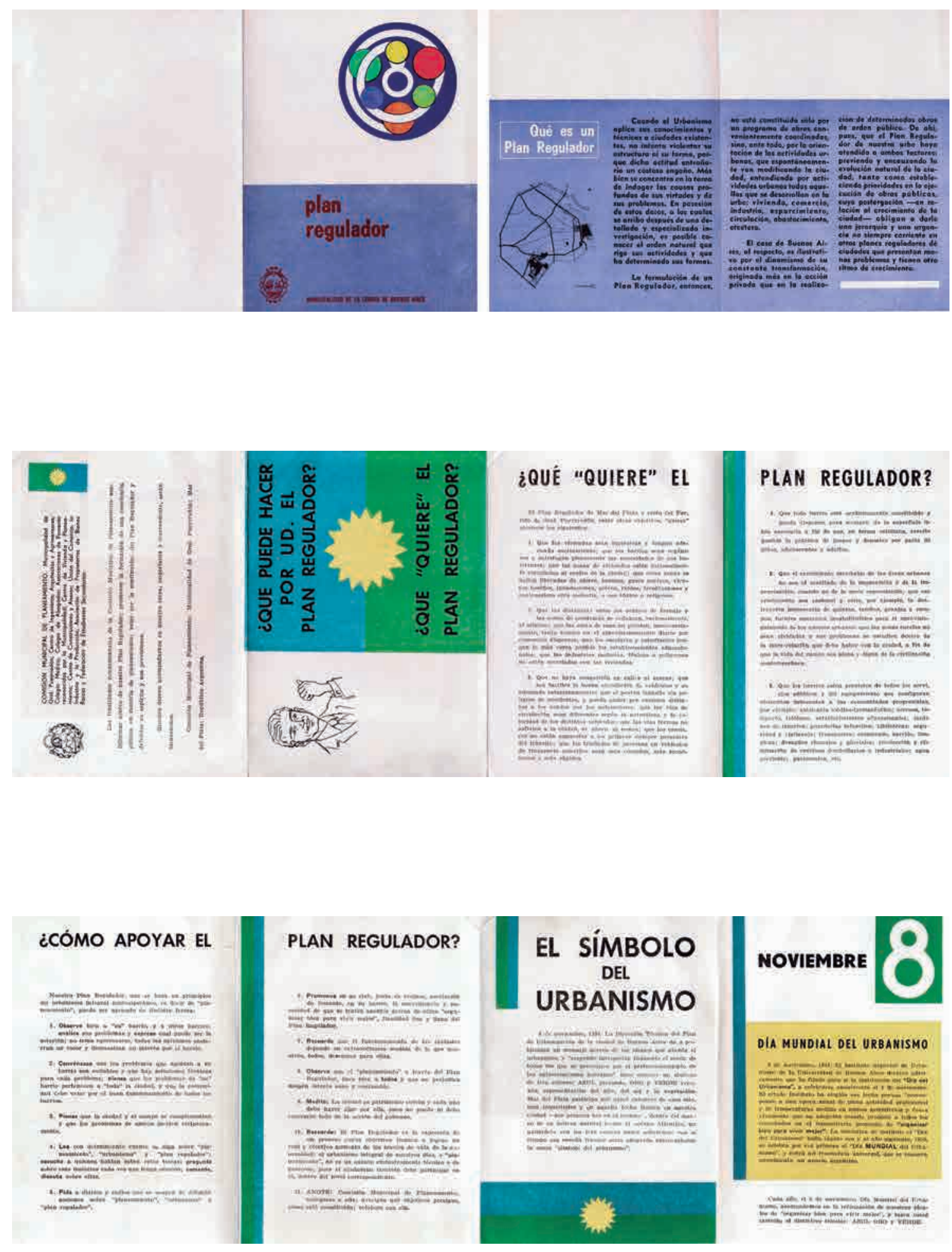

Figura 7, 8 y 9 Folletines sobre planeamiento, circa 1950. Fuente: Colección Pastor, Archivo del Instituto de Arte Americano FADUUBA 


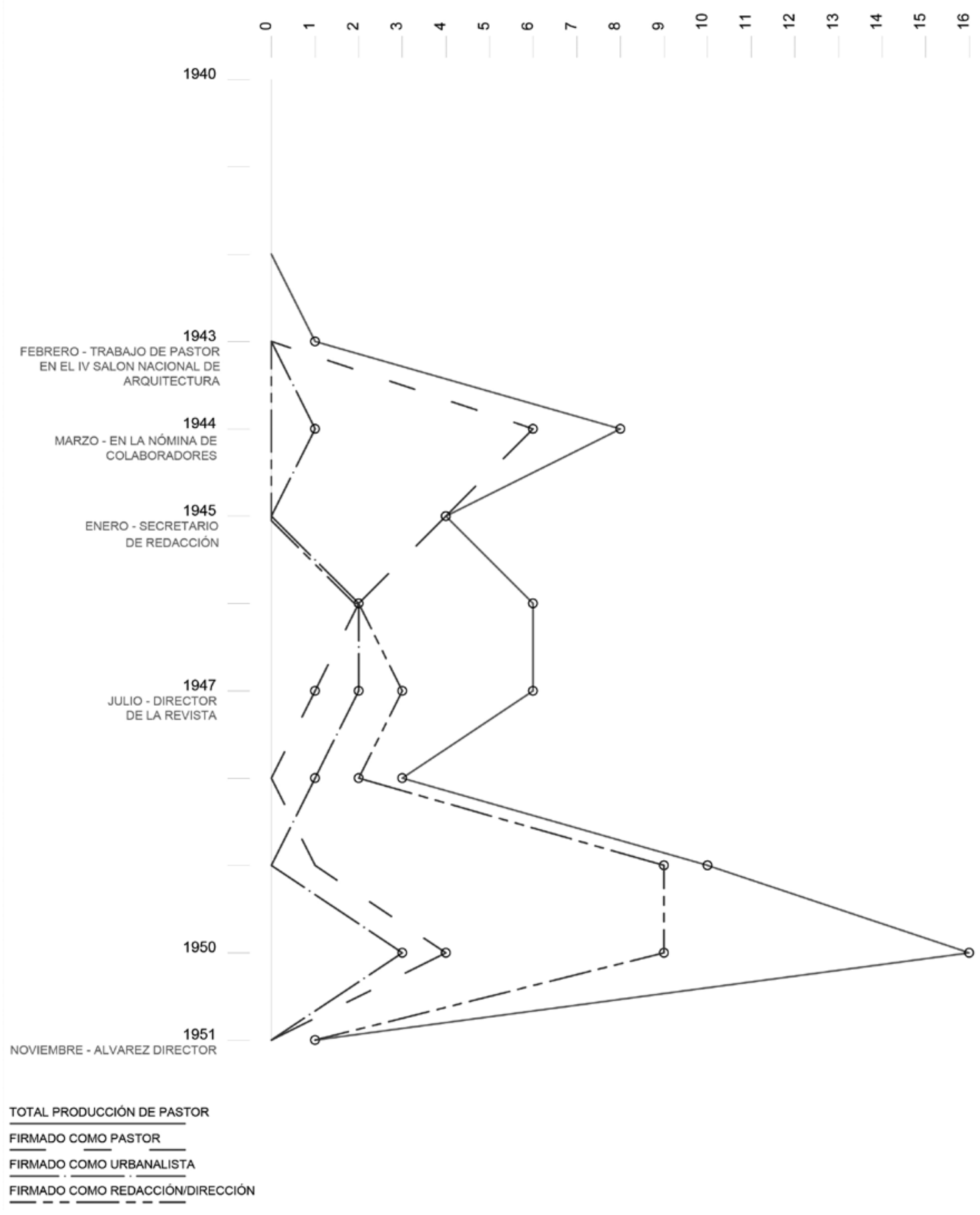

Tabla 1 La producción de Pastor en la Revista de Arquitectura de la Sociedad Central de Arquitectos. Fuente: Elaboración del autor. 
CONCLUSIONES

Las publicaciones seriadas, como fuente primaria, se presentan en su conjunto como un rico medio para analizar el proceso de pensamiento de autores e instituciones. En el caso particular de esta selección de textos, el trabajo recorrido no muestra un desarrollo lineal del discurso de Pastor en la RdeA. La periodización de su producción, según su relación con la institución, se correspondió a distintas versiones de su discurso. Hay dos claros movimientos: de la vivienda como centro de la discusión, a la vivienda como componente del planeamiento; y del planeamiento como teoría a explicar, al planeamiento como práctica a implementar. El otro aspecto a rescatar del desarrollo de la Revista apunta a sus propios cambios y apertura de espacios. Más allá de la modernización de su propuesta gráfica, es de rescatar la apertura de la sección Crónica, tanto por su contenido como por el espacio editorial que se brinda a numerosos autores. Como ya se dijo, se destaca este espacio abierto en la última etapa de Pastor en la Revista, no solo por su volumen sino por su discurso propagandístico del planeamiento y por la defensa de los planificadores como profesionales. Si bien no existe una asociación directa entre el hecho de que Pastor sea Director y la aparición de los artículos de este periodo no se puede ignorar la relación que existe entre la producción previa de Pastor y la temática de estos artículos.

Son numerosas las líneas de investigación que esta exploración abre. En un principio merece ser propuesto un futuro trabajo más detallado del contenido de los artículos de la etapa de Pastor como Director de la Revista. A su vez, los matices de sus propios artículos también suscitan un trabajo más exhaustivo; es necesario un estudio de la voz empleada en los diferentes subgrupos de su producción. Tal vez el subgrupo más importante que se podría construir viene dado por las firmas del arquitecto: Pastor, Urbanalista, redacción, dirección. Otra categoría a construir en el interior de este recorte surge de los referentes temáticos; quedará para un posterior trabajo indagar sobre el movimiento entre los referentes que Pastor toma y su propia producción intelectual.

El estudio de la literatura técnica producida por Pastor se complementa tanto con su bibliografía como con un importante caudal de producción gris (Figuras 7, 8 y 9), por ejemplo, folletines sobre urbanismo y planeamiento o las publicaciones periódicas del IPRU, el cual funcionó como organismo de divulgación y producción en la siguiente etapa de Pastor (1951 en adelante). Dicho conjunto de producción gris prolonga las líneas temáticas estudiadas en este artículo, de modo que es posible buscar allí continuidades y, a la vez, observar los cambios en la postura del autor.

\section{REFERENCIAS BIBLIOGRÁFICAS}

BRUNO, Paula. Paul Groussac. Un articulador cultural en el pasaje del siglo XIX al XX argentino. Araucaria, Revista Iberoamericana de Filosofía, Política y Humanidades, 2006, n8, pp. 176-186.

CIRVINI, Silvia Augusta. Las revistas técnicas y de arquitectura (1880-1945). Periodismo especializado y modernización en Argentina. Argos, 2011, n54, vol. 28, pp. 13-60.

Divulgación del urbanismo y la arquitectura. Revista de Arquitectura, octubre de 1949, año 34, n³46, pp. CCXCVII.

GENTILE, Eduardo. Revista de Arquitectura. En: Liernur, Jorge Francisco y Aliata, Francisco (comps.). Diccionario de arquitectura en la Argentina: estilos, obras, biografías, instituciones, ciudades. Buenos Aires: AGEA, 2004, pp. 175-178.

GORELIK, Adrián. Pastor, José M. F. En: Liernur, Jorge Francisco y Aliata, Francisco (comps.). Diccionario de arquitectura en la Argentina: estilos, obras, biografías, instituciones, ciudades. Buenos Aires: AGEA, 2004, pp. 46-48.

GUTIÉRREZ, Ramón. Sociedad Central de Arquitectos. 100 años de compromiso con el país 1886/1986. Buenos Aires: SCA, 1993.

MAZZA, Carlos Jerónimo y BRUNO, Peralta Ana. Políticas públicas para la organización del territorio en la provincia de Buenos Aires en la gobernación de Domingo Mercante. Ponencia presentada en el Primer Congreso de Estudios sobre el Peronismo: La Primera Década, Mar del Plata, 2008.

NOVICK, Alicia y PICCIONI, Raúl. Carlos María Della Paolera o la Amnesia del Urbanismo Argentino. Anales del Instituto de Arte Americano e Investigaciones Estéticas "Mario J. Buschiazzo", 1998, n³0, pp. 112-149.

PASTOR, José Manuel Felipe. El pasado, la arquitectura y el futuro. Revista de Arquitectura, enero 1944, año 29, n²77, pp. 20-21.

PASTOR, José Manuel Felipe y PRATS, Roque. Centro juvenil de recreación. Revista de Arquitectura, enero 1944, año $29, n^{\circ} 277$, pp. 36-43.

PASTOR, José Manuel Felipe. San Juan. Piedra de toque del planeamiento nacional. Primera edición. Buenos Aires: Editorial Arte y Técnica, 1945.

PASTOR, José Manuel Felipe. Urbanismo con planeamiento. Principio de una nueva técnica social. Buenos Aires: Editorial Arte y Técnica, 1947.

PASTOR, José Manuel Felipe. Curso básico de planeamiento urbano y rural. Buenos Aires: Ministerio de Obras Públicas. Provincia de Buenos Aires, 1950.

PASTOR, José Manuel Felipe. TVA. Planificación del Valle del Tennesse. Buenos Aires: Contempora, 1951.

Revista de Arquitectura cumple 25 años. Revista de Arquitectura, julio 1940, año 26, n² 235, pp. 193-194.

RIGOTTI, Ana María. Las invenciones del urbanismo en Argentina (1900-1960). Rosario: UNR Editora, 2014.

Una posta. Revista de Arquitectura, febrero 1943, año $28, \mathrm{n}^{\circ}$ 266, pp. 45-48. 